\title{
Größe der Pulmonalarterie korreliert mit Exazerbationen
}

\author{
Bei Patienten mit COPD ist eine schwere pulmonalarterielle \\ Hypertonie ein Prädiktor von Exazerbationen. Das Forscher- \\ team um J. M. Wells stellte nun die Hypothese auf, dass dies \\ für eine computertomografisch gemessene Vergrößerung \\ der Arteria pulmonalis ebenfalls gelten könnte. \\ N Engl J Med 2012; 367: 913-921
}

Die Autoren rekrutierten Personen im Alter zwischen 45 und 80 Jahren, die zum Studienzeitpunkt rauchten oder früher geraucht hatten und mindestens $10 \mathrm{~Pa}-$ ckungsjahre aufwiesen. Alle Teilnehmer unterzogen sich einer Spirometrie, einem 6-minütigen Gehtest sowie einem ThoraxCT und füllten zudem einen Fragebogen zu ihren Symptomen aus. In die Analyse gingen letztlich Patienten mit einer COPD im GOLD-Stadium II-IV ein, die dann im Median über 2,1 Jahre bezüglich ihres klinischen Verlaufs einschließlich der Exazerbationen beobachtet wurden. In den CTAufnahmen bestimmten die Autoren das
Verhältnis der Durchmesser von Pulmonalarterie und Aorta (PA/A) und überprüften, ob ein Verhältnis von mehr als 1 mit schweren Exazerbationen assoziiert war, die eine Hospitalisierung erforderlich machten. Die gleiche Analyse führten sie an einer externen Validierungskohorte aus Teilnehmern einer anderen Studie durch.

In die Auswertung gingen 3464 Patienten ein, die anhand des Verhältnisses PA/A in 2 Gruppe unterteilt wurden (PA/A $\leq 1$; $\mathrm{n}=2645$ und PA/A > 1; $\mathrm{n=819}$ ). Die multivariate Regressionsanalyse zeigte, dass bei Teilnehmern mit einem Verhältnis PA/A von mehr als 1 zum Zeitpunkt des Studienbeginns eine deutliche Korrelation mit schweren Exazerbationen mit einer Odds Ratio von 4,78 bestand. Auch im Studienverlauf war ein Verhältnis PA/A > 1 mit einem erhöhten Risiko für zukünftige schwere Exazerbationen assoziiert. Dies galt sowohl für die Studienkohorte als auch für die externe Validierungskohorte mit Odds Ratios von 3,44 bzw. 2,80.

In beiden Kohorten zeigte von allen analysierten Variablen ein PA/A-Verhältnis von mehr als 1 die stärkste Assoziation mit schweren Exazerbationen. Auch für jegliche Formen von Exazerbationen fand sich eine Korrelation mit dem PA/A-Verhältnis mit Odds Ratios von 1,86 in der Studienkohorte und 2,17 (1 Jahr) bzw. 6,68 (3 Jahre) in der Validierungskohorte.

\section{Fazit}

Eine im CT bestimmte Vergrößerung der Pulmonalarterie, ausgedrückt als ein Verhältnis der Durchmesser von Arteria pulmonalis und Aorta größer 1, war bei Patienten mit COPD mit schweren Exazerbationen assoziiert, so die Autoren.

\section{Dr. Johannes Weiß, Bad Kissingen}

„Mit MycPermCheck ist es möglich, die Suche nach Wirkstoffen gegen Mykobakterien auf genau die chemischen Verbindungen zu fokussieren, die mit hoher Wahrscheinlichkeit in das Bakterium eindringen können“, sagt Sotriffer. Damit ließen sich virtuelle und experimentelle Screenings effizienter und zielgerichteter durchführen. Mit dem Modell kann man auch prüfen, ob neu entworfene Substanzen die gewünschten Eigenschaften besitzen. „Ist das nicht der Fall, kann versucht werden, dies durch ein gezieltes molekulares Design zu erreichen“, so Sotriffer. Jedoch ist MycPermCheck kein Verfahren, mit dem sich die Durchlässigkeit der bakteriellen Zellwand exakt berechnen lässt.

MycPermCheck lässt sich als kostenloser Webservice auf der Homepage der Arbeitsgruppe Sotriffer frei nutzen: www.mycpermcheck.aksotriffer.pharmazie.uniwuerzburg.de

Nach einer Pressemitteilung der JuliusMaximilians-Universität Würzburg 